\title{
Automatic Decision Maling of Washing the Rapid Polluted Insulators in Substations
}

\author{
¿Satoru GOTO†, Masatoshi NAKAMURA†, Nishantha NANAYAKKARA†, \\ Tatsuhide MATSUNAGA $\dagger$ and Takashi TANIGUCHI
}

$\dagger$ †epartment of Electrical Engineering, Saga University, Honjomachi, Saga 840, Japan \$Saga Branch, Kyushu Electric Power Co., Ltd., 2-3-6, Konohigashi, Saga 840, Japan

\begin{abstract}
This study is aimed to develop an automatic decision making of the time of washing the polluted insulators in substations. The factors of the automatic decision making are determined by the right state of the information of an expert's decision making. The automatic decision is made based on the index of the linear discriminant function. The proposed method is applied to the actual data of a substation and assured the usefulness. The proposed method accomplishes the role of the human decision making, automatically.
\end{abstract}

\section{Introduction}

The insulators in substations play the role of isolating the live conductors from the ground potential. The insulators in the coastal areas are polluted by the wind with much content of salt. The insulator pollution is a big problem which causes flash over of the substation equipments and result power failures. The insulator pollution problem was analyzed[1][2] and the prediction of the pollution rate has been proposed earlier by authors [3]. The polluted insulators are usually washed by fresh water before a dielectric breakdown occurs, following the decision made by experts.

However, this kind of human decision is not always correct and there is no exact rule for the decision making. The decision was made based on the trade off between the cost of the washing and the emergency level of the substation. Ineffective washing must be avoided and an appropriate decision making is required to reduce the overall cost for the insulator washing.

This study is aimed to develop an automatic decision making of the time of washing the polluted insulators in substations, which accomplishes the role of the human decision making, automatically.

\section{Right State of Decision Making of Washing the Polluted Insulators}

\subsection{Data Acquisition}

Actual data of insulator pollution, decision making of washing and weather conditions were acquired during the periods of September 1st 1991 to October 31st 1991 and
September 1st 1992 to December 31st 1992 at Karatsu substations of Saga prefecture in Japan. The available data are pollution deposits $\left[\mathrm{mg} / \mathrm{cm}^{2}\right]$, wind velocity $[\mathrm{m} / \mathrm{s}]$, wind direction ( 16 directions), rainfall $[\mathrm{mm} / \mathrm{h}]$, temperature $\left[{ }^{\circ} \mathrm{C}\right]$, humidity[\%] and sea level[mm].

The pollution deposits of the substation insulators were measured by using a pilot insulator and a salt meter. The leakage current of the pilot insulator is measured at a selected D.C. voltage $(10[\mathrm{kV}])$ and a selected humidity level $(70[\%])$. Afterwards, the corresponding pollution deposit is determined from the standard graph of the leakage current v.s. the pollution deposit. The pilot insulator is placed near the existing insulators of the substation. At first the pilot insulator is immersed in the salt meter and by using vapor the humidity level in the containment is raised up to $70[\%]$, to have a sufficient wet insulator. After that $10[\mathrm{kV}]$ D.C. voltage is applied across the electrodes of the pilot insulator and the leakage current is measured. The corresponding pollution deposit $\left[\mathrm{mg} / \mathrm{cm}^{2}\right]$ is selected from the available standard plots. After having finished the process, the insulator is dried and lifted it out from the salt meter and positioned at the same height of other insulators. The entire process takes approximately 30 minutes. Despite some small errors in the measurements due to the nonuniform distribution of pollution deposits and no effect of pollution during the period in the compartment of the salt meter, this method is widely adopted to get the pollution deposits of the insulators because there is no other reliable method to get the pollution deposit data of the substation insulators. 
The pollution deposits of the insulators is usually measured every 3 hours. However, at the times of typhoons, the rapid pollution would occur and the measurement interval is shorten to one hour.

Figure 1 shows a part of the actual data of the insulator pollution and the weather conditions at the Karatsu substation during October 1991. In the pollution graph of the Fig. 1, the symbol + means the decision making of the washing. As shown in the figure, the rapid pollution occurred around 11th October 1991 and the measurement interval of the pollution deposits was shorten to one hour, when the typhoon 21 passed through the substation. The strong wind comes from the north direction without any rainfall is the most dangerous for the insulator pollution at the Karatsu substation. In the Fig. 1, it is also found that the pollution deposits were decreased by the insulator washing:

\subsection{Causes of the Pollution}

According to the analysis of the collected data, we found a relationship between the pollution and the weather conditions. Wind velocity, wind direction and rainfall were found to be main factors for the pollution. Strong wind velocity causes the faster pollution of the insulators. Wind direction is also related to the pollution because the wind from the seashore contains much salt. The rain has the natural washing of the insulators and, in turn decreases of the pollution. Especially, the rapid pollution of the insulators occurs in bad weather conditions at typhoons. Typhoons without any rainfall are the most dangerous for the insulator pollution. The atmosphere temperature, humidity and sea level have few effects to the pollution of the insulators.

\subsection{Decision Making of Insulator Washing}

An expert makes the decision of the insulator washing based on one's experience and measurement data in the Karatsu substation. The information of the decision making are the pollution deposits, weather information (wind velocity, wind direction, rainfall and typhoon information) and the noise of the leakage. The pollution deposit is the main factor to decide the insulator washing. When the pollution deposit is over the threshold value $\left(0.007\left[\mathrm{mg} / \mathrm{cm}^{2}\right]\right.$ at the Karatsu substation), the insulators are decided to be washed. If the pollution deposit becomes hazard level $\left(0.06\left[\mathrm{mg} / \mathrm{cm}^{2}\right]\right.$ at the Karatsu substation $)$, the operations of the substation are stopped and then the insulators are washed. Such situation causes the long power failures and it must be avoided. At weekend, the pollution deposit in one or two days ahead is predicted by the expert based on the weather information, and if the predicted pollution deposit is over the threshold value, the insulator washing is carried out at the weekend.

\section{Automatic Decision Making of Insulator Washing}

\subsection{Factors of Automatic Decision Making}

The factors of the automatic decision making of washing polluted insulators in the substation are determined based on the information of the expert's decision making. The factors of the automatic decision making are pollution deposit, pollution rate, wind velocity, wind direction, rainfall and the day is a Friday or not. The pollution deposit is the main factor of the decision making. The pollution rate, wind velocity, wind direction and rainfall give the prediction of the pollution deposit. A Friday is adopted by corresponding to the weekend washing. By using the above factors, the automatic decision making function is defined.

\subsection{Automatic Decision Making by Use of Linear Discriminant Function}

We adopted the linear discriminant function[4] for the index of the automatic decision making. The index $Z$ is defined by the linear combination of the factors $X_{i},(i=$ $1, \cdots, k)$ as

$$
Z=w_{1} X_{1}+w_{2} X_{2}+\cdots+w_{k} X_{k}
$$

where $w_{i}(i=1, \cdots, k)$ are the parameters and $k$ is the number of the factors. The parameters $w_{i}(i=1, \cdots, k)$ are determined to maximize the distance function $G$ between the index $z_{1}$ for the group 1 and the index $z_{2}$ for the group 2. The distance function $G$ is defined as

$$
G=\frac{\left(\bar{z}_{1}-\bar{z}_{2}\right)^{2}}{\sum_{i=1}^{2} \sum_{j=1}^{n_{i}}\left(z_{i j}-\bar{z}_{i}\right)^{2}}
$$

where $n_{i}$ is the number of the samples in the $i$ th group, $z_{i j}$ is the value of the index $Z$ for the $j$ th sample in the $i$ th group and $\bar{z}_{i}$ is the average value of the index $Z$ for the $i$ th group.

The partial differential of the distance function $G$ with respect to the parameters $w_{i}(i=1, \cdots, k)$ leads the linear equations as

$$
w_{1} S_{r 1}+w_{2} S_{r 2}+\cdots+w_{k} S_{r k}=d_{r}, \quad r=1, \cdots, k
$$

where

$$
\begin{aligned}
S_{p q} & =\sum_{i=1}^{2} \sum_{j=1}^{n_{i}}\left(x_{p i j}-\bar{x}_{p i}\right)\left(x_{q i j}-\bar{x}_{q i}\right) \\
d_{p} & =\bar{x}_{p 1}-\bar{x}_{p 2}
\end{aligned}
$$

$x_{p i j}$ is $i$ th factor of the $j$ th sample in the $p$ th group and $\bar{x}_{p i}$ is the average of the $x_{p i j}$ in the $i$ th group. By solving the linear equations (3), the parameters $w_{i}(i=1, \cdots, k)$ are calculated.

The factors of the linear discriminant function are pollution deposit $\left(x_{1}\right)$, pollution rate $\left(x_{2}\right)$, equivalent wind velocity $\left(x_{3}\right)$, rainfall $\left(x_{4}\right)$ and the day is a Friday or not 


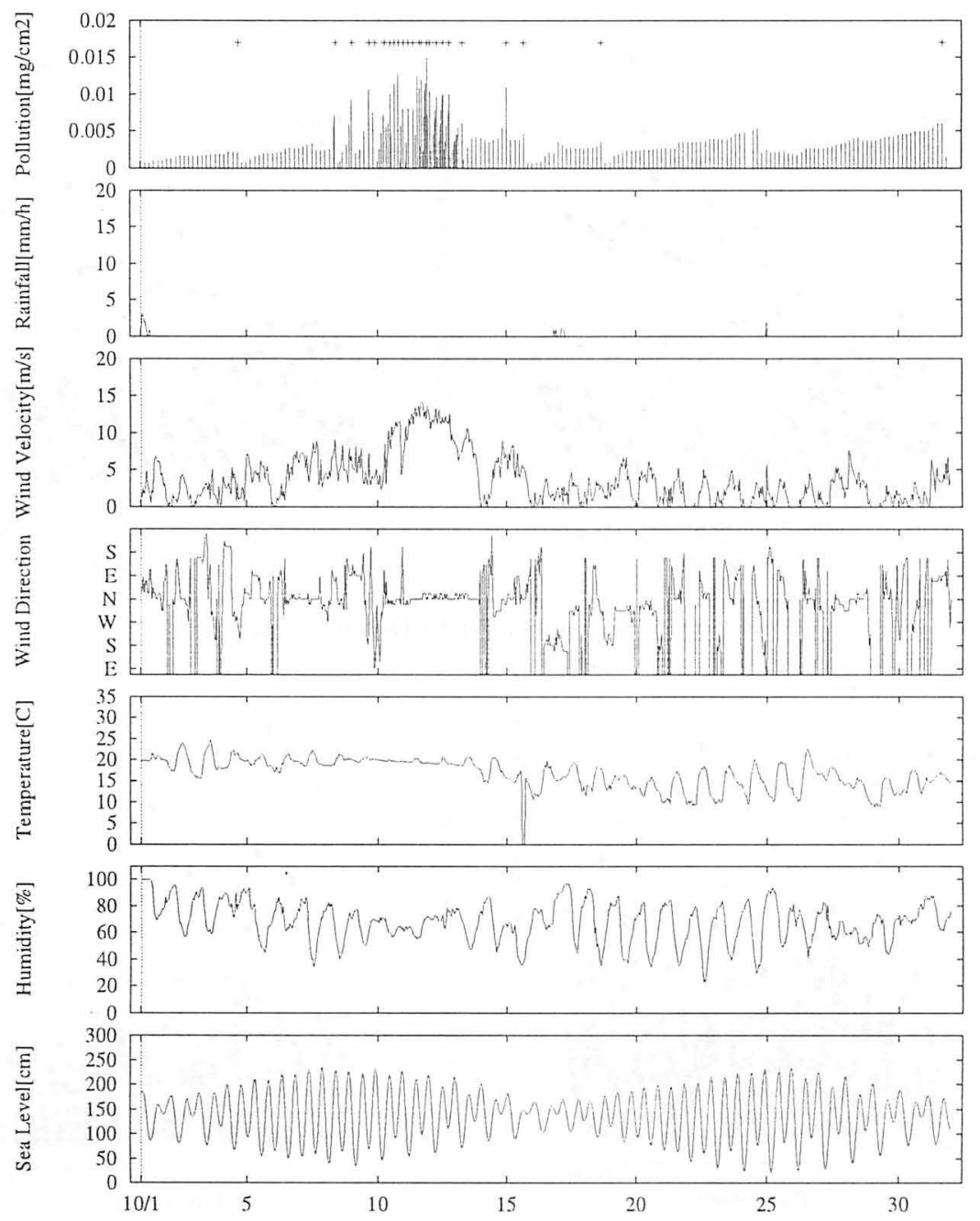

Fig. 1. Actual data of the insulator pollution, the decision making of washing and the weather information at Karatsu substation during October 1991.

$\left(x_{5}\right)$. Here, the equivalent wind velocity is the weighted wind velocity according to the wind direction. The weights are determined by considering the affection to the pollusion deposits as 1 for $\mathrm{N}-\mathrm{NE}, 0.79$ for ENE-E, 0.40 for ESE-SW and 0.57 for WSW to NNW[3].

Automatic decision making of washing is implemented if the following conditions are fulfilled

$$
\begin{cases}\text { Washing } & z>\lambda \\ \text { No washing } & z \leq \lambda,\end{cases}
$$

where $\lambda$ was the threshold value of the automatic decision making. The threshold value $\lambda$ is determined such that the sum of the successful percentage of the both decisions of the washing and the no washing is maximized.

\subsection{Applications to Actual Data}

The effectiveness of the automatic decision making was assured based on the actual data. We divided the insulator pollution data into three groups as follows:

- Group A: Insulator washing is decided.

- Group B: One step before the data of the Group A.

- Group C: Otherwise.

The data in Group B are ignored for the automatic decision making and the data in group $A$ and tha data in group $C$ are distinguished because the data in group $B$ are near the data in Group $A$ and the expert wavers in the judgment of the washing.

The parameters for the factors in (1) are determined by using the collected data at the Karatsu substation during September 1st to October 31st 1991 as $w_{1}=4.2$, $w_{2}=0.2, w_{3}=0.0003, w_{4}=0.0003$ and $w_{5}=0.0058$, and the threshold value is detemined as $\lambda=0.0208$. Table 1 shows the result of the automatic lecision making compared with the actual decision making during September 1st to October 31st 1991 at the Karatsu substation. 
(a) Period of September 1st to October 31st 1991
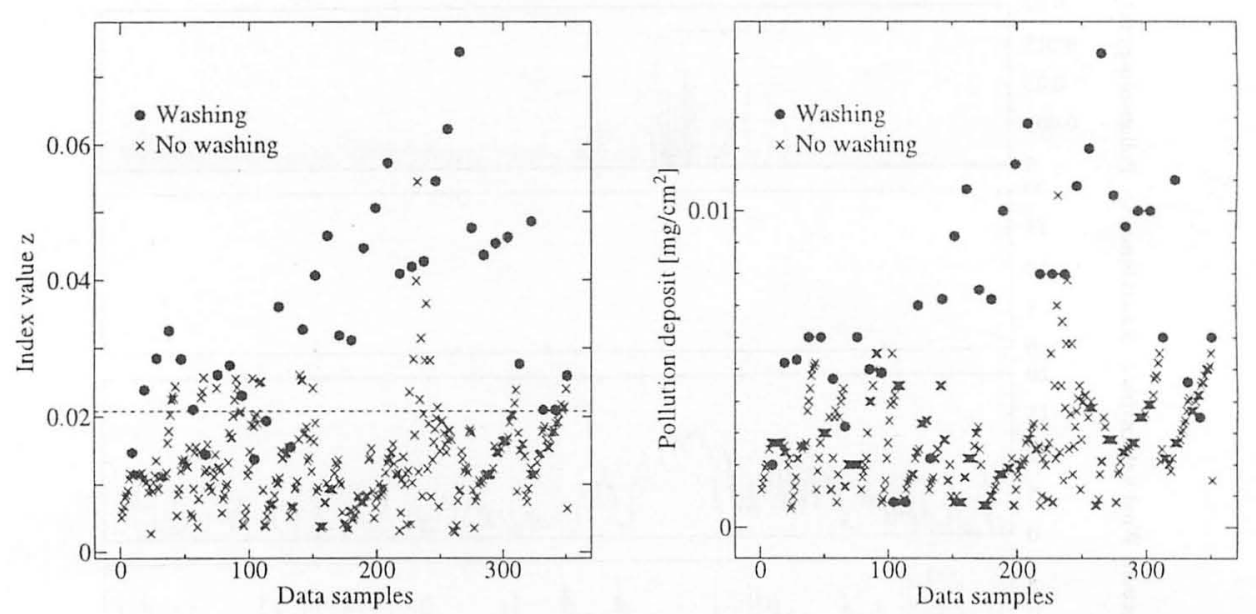

(b) Period of September 1st to December 31st 1992
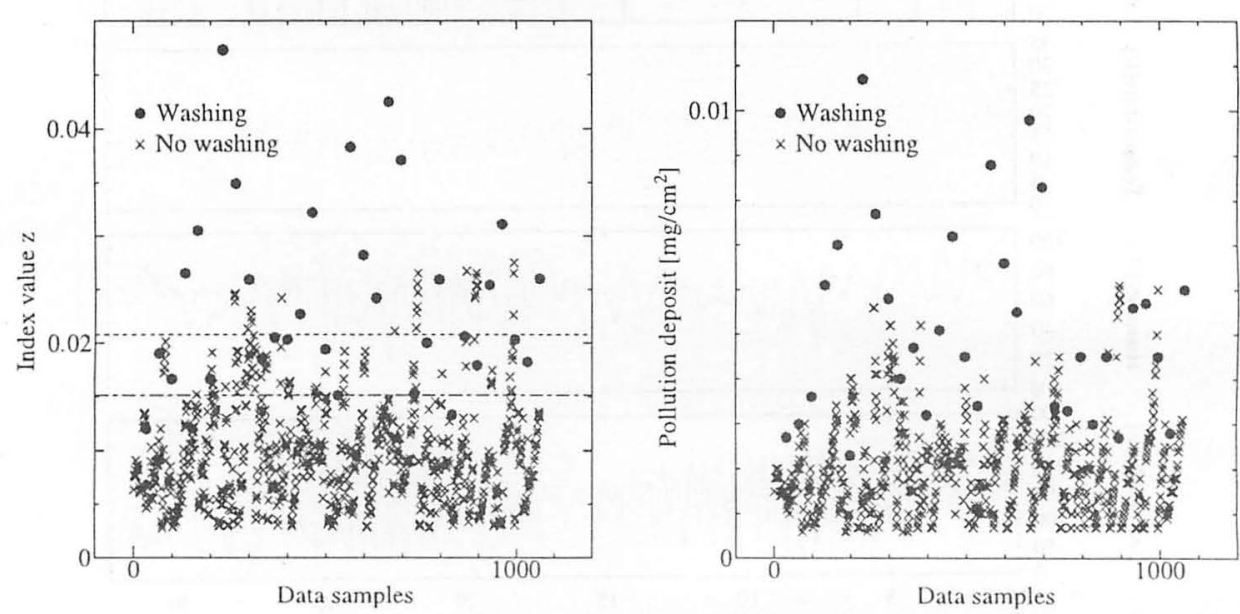

Fig. 2. Index value $z$ and pollution deposits at Karatsu substation.

Table 1 Automatic decision making of washing at Karatsu substation during September 1st to October 31st $1991(\lambda=$ $0.0208)$

\begin{tabular}{|c|c|c|c|}
\hline Data & Total & Success & Fault \\
\hline Washing & 37 & $32(86.5 \%)$ & $5(13.5 \%)$ \\
\hline No washing & 351 & $314(89.5 \%)$ & $37(10.5 \%)$ \\
\hline
\end{tabular}

Over $85[\%]$ is successful at automatic decision making and the result shows the usefulness of the proposed method. The 5 data of the washing data could not be detected by the proposed method. The days of the 4 data in the 5 fault data were Fridays and in the 2 data, the typhoon 19 passed through the Karatsu substation and the decisions were made for safety side despite of the small pollution de- posits, i.e. $0.0008\left[\mathrm{mg} / \mathrm{cm}^{2}\right]$. In the last data, the typhoon 17 passed through the Karatsu substation and the decision was also made for safety side despite of the low pollution deposits, i.e., $0.0022\left[\mathrm{mg} / \mathrm{cm}^{2}\right]$.

Data collected from the Karatsu substation during September 1st to December 31st 1992 were used for the validation of the proposed method. Table 2 shows the validation result by using the same parameters and the same threshold value determined by the data collected during September 1st to October 31st 1991. The automatic decision making of the washing is not good becanse of the bad threshold value $\lambda=0.0208$ for the data during September 1st to December 31st 1992. It is caused by the rapid pollution in the data during September 1st to October 31st 1991 and the increase of the threshold value. The result by using the optimal threshold value $\lambda=0.0151$ for the data during 
Table 2 Automatic decision making of washing at Karatsu substation during September 1st to December 31 st 1992 $(\lambda=0.0208)$

\begin{tabular}{|c|c|c|c|}
\hline Data & Total & Success & Fault \\
\hline Washing & 32 & $16(50.0 \%)$ & $16(50.0 \%)$ \\
\hline No washing & 1062 & $1034(97.4 \%)$ & $28(2.6 \%)$ \\
\hline
\end{tabular}

Table 3 Automatic decision making of washing at Karatsu substation during September 1st to December 31st 1992 (The threshold is modified to $\lambda=0.0151$ )

\begin{tabular}{|c|c|c|c|}
\hline Data & Total & Success & Fault \\
\hline Washing & 32 & $30(93.8 \%)$ & $2(6.2 \%)$ \\
\hline No washing & 1062 & $943(88.8 \%)$ & $119(11.2 \%)$ \\
\hline
\end{tabular}

September 1st to December 31st 1992 is shown in table 3. The result in table 3 is much better than that in table 2 and the suitable threshold value is very important for the automatic decision making of the insulator washing. The 2 data of the washing data could not be detected by the proposed method. The days of the 2 data were not corresponding to the weekend washing. However, the pollution deposits of the 2 data were small, i.e., $0.0027\left[\mathrm{mg} / \mathrm{cm}^{2}\right]$ and $0.0030\left[\mathrm{mg} / \mathrm{cm}^{2}\right]$. Is is not serious failure that the proposed method could not detect these 2 data.

The proposed method is compared with the pollution deposit method, which means that the decision is made by only the pollution deposit. Figure 2 shows the index value $z$ and the pollution deposits. As shown in Fig. 2, the index value $z$ is better than the pollution deposit. The distance function $G$ is calculated for each method. By using the proposed method, $G=2.22 \times 10^{-2}$ for the data during September 1st to October 31st 1991 and $G=8.74 \times 10^{-3}$ for the data during September 1st to December 31 st 1992 and by using the pollution deposit method, $G=1.91 \times$ $10^{-2}$ for the data during September 1st to October 31st 1991 and $G=8.69 \times 10^{-3}$ for the data during September 1st to December 31st 1992. For each data, the distance function by using the proposed method is bigger than that by using the pollution deposit method. The results show the effectiveness of the proposed method compared with the pollution deposit method.

\section{Conchusions}

An automatic decision making of the time of washing the polluted insulators in substations is proposed. The automatic decision making function is derived by the linear discriminant function. The proposed method is applied to the actual data of the Karatsu substation and over 85[\%] of human decisions were correctly detected automatically with the proposed automatic decision making process. By using the automatic decision making, the suitable decision making of the washing could be done withont any expert's works.

\section{References}

[1] Electric Joint Research, vol. 20, no, 2 (1964) (in Japanese)

[2] Electric Joint Research, vol. 35, no. 3 (1979) (in Japanese).

[3] N. Nanayakkara, M. Nakamura, S. Goto and T. Taniguchi: Modeling and Prediction of Rapid Pollution of Insulators in Substations Based on Weather Information, Proceedings of the 19.94 liorean Automatic Control Conference (1994)

[4] D. G. Hoel: Introduction to Mathematical Statistics. 4th ed., John Wiley \& Sons, Inc. (1971). 
